\title{
Circulating exosomal mRNA profiling identifies novel signatures for the detection of prostate cancer
}

\author{
Jin $\mathrm{Ji}^{1 \dagger}$, Rui Chen ${ }^{1 \dagger}$, Lin Zhao ${ }^{1 \dagger}$, Yalong $\mathrm{Xu}^{1 \dagger}$, Zhi Cao ${ }^{1}$, Huan Xu${ }^{1}, \mathrm{Xi}$ Chen ${ }^{1}$, Xiaolei Shi ${ }^{1}$, Yasheng Zhu', Ji Lyu ${ }^{1,2}$, \\ Junfeng Jiang ${ }^{3}$, Yue Wang ${ }^{3}$, Tie Zhou' ${ }^{1}$ Jingyi He${ }^{4}$, Xuedong $\mathrm{Wei}^{4}$, Jason Boyang $\mathrm{Wu}^{5}$, Bo Yang ${ }^{1 *}$ and \\ Fubo Wang ${ }^{1 *}$ (iD
}

\begin{abstract}
The landscape and characteristics of circulating exosomal messenger RNAs (emRNAs) are poorly understood, which hampered the accurate detection of circulating emRNAs. Through comparing RNA sequencing data of circulating exosomes with the corresponding data in tissues, we illustrated the different characteristics of emRNAs compared to tissue mRNAs. We then developed an improved strategy for emRNA detection based on the features of circulating emRNAs. Using the optimized detection strategy, we further validated prostate cancer (PCa) associated emRNAs discovered by emRNA-seq in a large cohort of patients and identified emRNA signatures for PCa screening and diagnosis using logistic regression analysis. The receiver operating characteristic curve (ROC) analysis showed that the circulating emRNA-based screening signature yielded an area under the ROC curve (AUC) of 0.948 in distinguishing PCa patients from healthy controls. The circulating emRNA-based diagnostic signature also showed a great performance in predicting prostate biopsy results (AUC: 0.851). In conclusion, our study developed an optimized emRNA detection strategy and identified novel emRNA signatures for the detection of PCa.
\end{abstract}

Keywords: Exosome, Prostate cancer, RNA-sequencing, Diagnosis

\section{Main text}

Prostate cancer $(\mathrm{PCa})$ is the leading malignancy in Western men, with 1,276,106 new cases and 358,989 deaths in 2018 globally [1]. The accurate early detection of PCa is one of the key issues in the management of PCa. The early diagnosis of $\mathrm{PCa}$ depends on prostate-specific antigen (PSA) test-initiated prostate biopsy. However, the wide use of PSA has resulted in a number of unnecessary biopsies accompanying complications due to its low specificity. In addition, a previous study showed that

\footnotetext{
*Correspondence: yangbochanghai@126.com; wangbofengye@163.com ${ }^{\dagger}$ Jin Ji, Rui Chen, Lin Zhao and Yalong Xu contributed equally to this work. 'Department of Urology, Shanghai Changhai Hospital, Naval Medical University (Second Military Medical University), Shanghai, China Full list of author information is available at the end of the article
}

quite a few $\mathrm{PCa}$ cases, including high-grade $\mathrm{PCa}$ (HGPCa) cases, are missed since they can show PSA levels in the normal range [2]. Therefore, there is an urgent need to identify novel biomarkers with higher accuracy for the early detection of PCa.

Recent years have witnessed the promising roles of exosomal RNAs (exRNAs) in cancer detection [3]. Extracellular long RNAs (exLR), mainly messenger RNAs (mRNAs), could be potential biomarkers in glioma [4], hepatocellular carcinoma (HCC) [5], pancreatic ductal adenocarcinoma (PDAC) [6], etc. However, the landscape and characteristics of circulating exosomal mRNAs (emRNAs) are poorly understood [7], which hinders the accurate detection of emRNAs. Although

(c) The Author(s). 2021 Open Access This article is licensed under a Creative Commons Attribution 4.0 International License, which permits use, sharing, adaptation, distribution and reproduction in any medium or format, as long as you give appropriate credit to the original author(s) and the source, provide a link to the Creative Commons licence, and indicate if changes were made. The images or other third party material in this article are included in the article's Creative Commons licence, unless indicated otherwise in a credit line to the material. If material is not included in the article's Creative Commons licence and your intended use is not permitted by statutory regulation or exceeds the permitted use, you will need to obtain permission directly from the copyright holder. To view a copy of this licence, visit http://creativecommons.org/licenses/by/4.0/ The Creative Commons Public Domain Dedication waiver (http://creativecommons.org/publicdomain/zero/1.0/) applies to the data made available in this article, unless otherwise stated in a credit line to the data. 


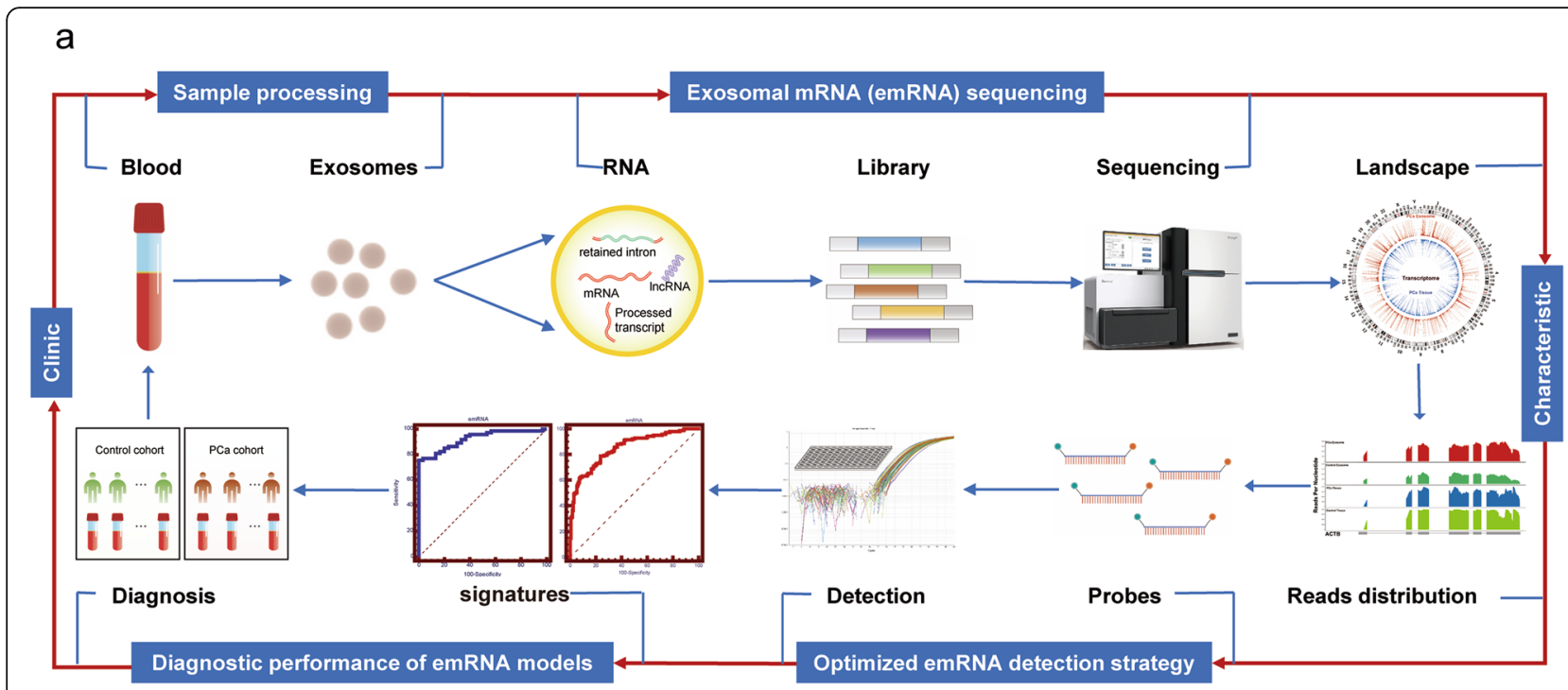

b

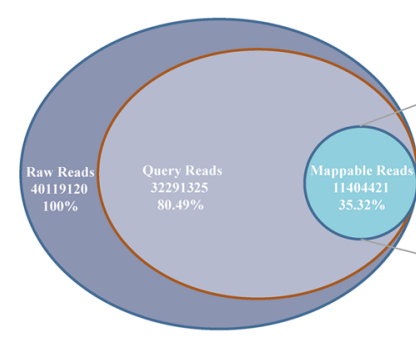

e

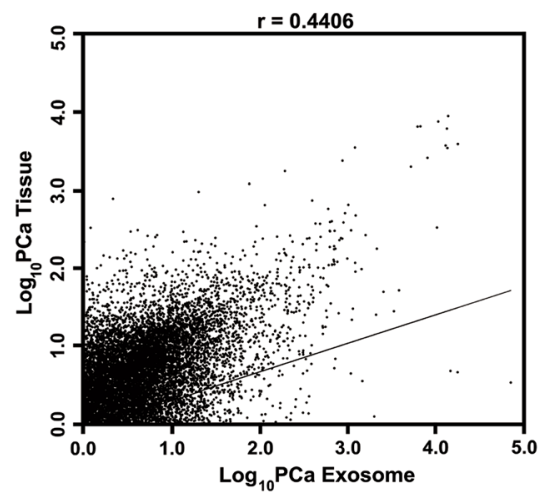

C

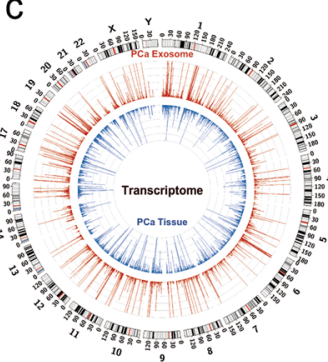

d

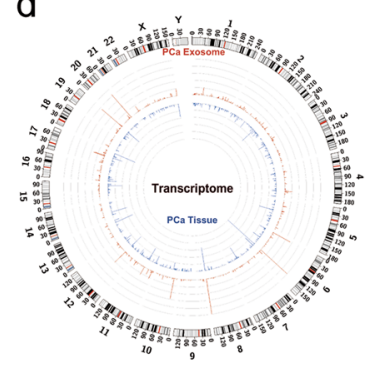

$f$
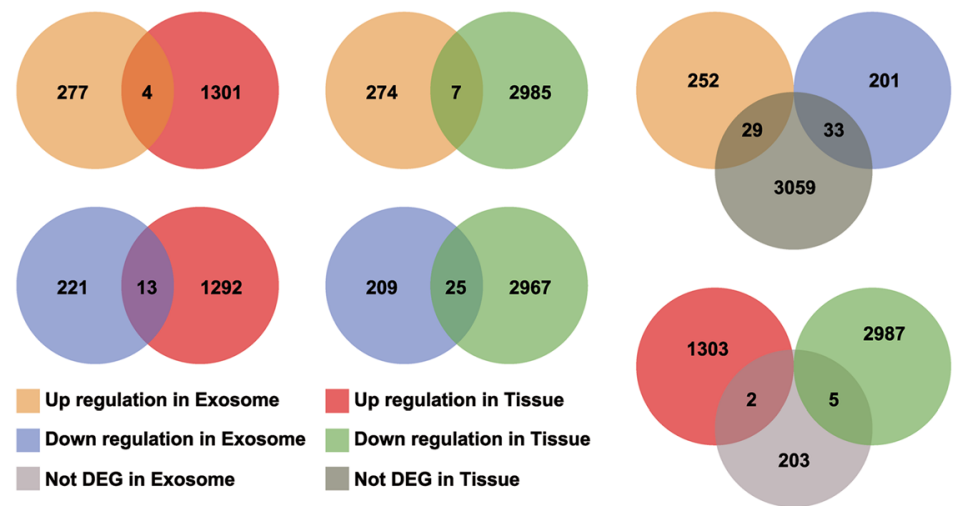

Fig. 1 Characterization of circulating exosomal mRNAs (emRNAs). a, Workflow of the study, including sample processing, emRNA sequencing, demonstrating the landscape and characteristics of emRNA, optimizing the detection strategy, and identifying tumor-specific emRNA signatures. b, The type and distribution of RNAs in circulating exosomes. Raw reads are the sequences detected by RNA sequencing. Query reads are those after trimming. Mappable reads are those mapped to known human RNA or genomes. Circos plots showing all mRNAs (c), and oncogene mRNAs (d), from PCa tissues and circulating exosomes of the same cohort of patients. e, Scatter plot illustrating the correlation between tissue mRNA and emRNA levels. $\mathbf{f}$, Venn diagrams showing the distinctive expression patterns between emRNAs and tissue mRNAs (based on the threshold of $p$ value $<0.05$ and fold change $>2$ for upregulated and fold change $<0.5$ for downregulated)

there have been reports on the use of extracellular microRNAs $[8,9]$ and an extracellular three-gene panel $[10,11]$ to detect PCa and HGPCa early, no studies have focused on the diagnostic potential of circulating
emRNAs. Here, we performed comprehensive sequencing of $\mathrm{PCa}$-associated emRNAs, developed an optimized detection strategy for emRNAs and established novel emRNA-based signatures for the detection of PCa. 


\section{The landscape and characteristics of circulating emRNAs}

To better understand the potential role of emRNA in PCa detection, the landscape and characteristics of circulating emRNAs were first illustrated. The workflow of the study is summarized in Fig. 1a (see details of Study design in Additional file 1). The quality control of exosome isolation and verification is shown in Additional file 1: Figure S1. After analyzing the emRNA profiles in $31 \mathrm{PCa}$ patients and $17 \mathrm{BPH}$ (benign prostatic hyperplasia) individuals (negative prostate biopsy) by RNAsequencing, we found that mRNA was the most abundant RNA, which was consistent with the findings of previous studies [5, 12] (Fig. 1b). We then compared the RNA sequencing data of circulating exosomes with the corresponding data in tissues. We mapped all mRNAs and mRNAs of oncogenes to the whole transcriptome of circulating exosomes and their corresponding tissues. The results indicated an overall even distribution of both mRNAs (Fig. 1c) and mRNAs of oncogenes (Fig. 1d) across the transcriptome between $\mathrm{PCa}$ tissues and exosomes. In addition, tissue mRNA levels were significantly correlated with emRNA levels $(r=0.441$, Fig. 1e). These results showed that circulating emRNAs could reflect the tissue mRNA profiles, providing a promising noninvasive method for cancer diagnosis.

We further compared the expression levels of PCaassociated tissue mRNAs and circulating emRNAs. A total of 4 mRNAs were identified to be upregulated and 25 mRNAs were identified to be downregulated in both emRNAs and tissue mRNAs in PCa patients. Seven mRNAs were upregulated in circulating exosomes but downregulated in tissue, and 13 mRNAs were downregulated in circulating exosomes but upregulated in prostate tissue in PCa patients (Additional file 1: Table S1 and Fig. 1f). These results demonstrated that there were distinctive expression patterns between emRNAs and tissue mRNAs, making circulating emRNAs more attractive and usable as a noninvasive biomarker for PCa diagnosis.

\section{An improved strategy for emRNA detection}

There is currently no consensus about the existing forms of emRNAs in peripheral blood, resulting in the inaccuracy and inconsistency of emRNA detection. A previous sequencing study estimated that most emRNAs appeared as shorter variants and fragments in the blood [5], which added to the difficulties in detection. Therefore, we first answered the question of whether circulating emRNAs were intact or presented as short variants before emRNA detection. We used Integrative Genomics Viewer (IGV) to visualize the reads distribution across the transcriptome of circulating emRNAs and tissue mRNAs. We compared the expression levels of each exon of candidate mRNA in circulating exosomes and in tissue and identified more variants in emRNAs than in tissue mRNAs
(Additional file 1: Figure s2 and s4b-c, see details of Identification the existing forms of circulating emRNAs in Additional file 1). Therefore, we developed an optimized detection strategy for emRNAs as follows. First, we mapped the read density of each candidate mRNA across the transcriptome in circulating exosomes and in tissue to estimate the abundance of these variants. We designed multiple primers for different regions in the exons and validated the primers by reverse transcription polymerase chain reaction (RT-PCR) (Primers are listed in Additional file 1: Table S2). Then, the detectable and unique bands were chosen as the targeted amplicon of candidate emRNAs. Primers for RT-PCR and TaqMan probes for quantitative PCR (qPCR) were designed accordingly.

\section{EmRNA signatures for the detection of PCa}

We identified PCa-associated emRNAs by RNAsequencing. Representative upregulated $(p<0.05$, fold change $>2)$ and downregulated $(p<0.05$, fold change $<$ $0.5)$ emRNAs are shown in Fig. 2a. We further evaluated the diagnostic performance of the $\mathrm{PCa}$-associated emRNAs. The workflow is summarized in Fig. 2b. First, 281 upregulated emRNAs in PCa were identified (Additional file 1: Table S3). We then identified a total of 9 emRNAs (TXK, ATM, TOX4, MAX, STK4, GRK5, PDGFA, RASS $F 5$, and IL32) with diagnostic potential for the detection of PCa by least absolute shrinkage and selection operator (LASSO) regression analysis (Additional file 1: Figure S3). Another 4 top upregulated emRNAs (CDC42, $F A M 228 B, N C F 2$ and SRSF2) according to the emRNAseq results were also selected for further testing. The optimized detection strategy developed in this study was applied to evaluate the expression of the 13 emRNAs candidates (Additional file 1: Figure S4, see details of Optimized detection strategy for the detection of 13 PCa-associated emRNAs in Additional File 1). After testing in 10 pairs of PCa patients and controls, 3 emRNAs (TXK, ATM and TOX4) were excluded because they showed no difference between PCa patients and controls or showed inconsistencies with the RNA-seq results (Additional file 1: Figure S5a-f), and the remaining 10 detectable emRNAs (MAX, STK4, GRK5, PDGFA, IL32, RASSF5, CDC42, FAM228B, NCF2 and SRSF2) were included for further validation. After evaluating the expression levels in $76 \mathrm{PCa}$ patients and $84 \mathrm{BPH}, 4$ emRNAs (STK4, GRK5, RASSF5 and FAM228B) were excluded because they showed no difference between PCa patients and controls (Additional file 1: Figure S5gj). Finally, 6 upregulated emRNAs (CDC42, IL32, MAX, NCF2, PDGFA and SRSF2) were finally confirmed in 141 $\mathrm{PCa}$ patients, $170 \mathrm{BPH}$ patients with negative prostate biopsy and 30 healthy controls. As shown in Fig. 2c, CDC42, IL32, MAX, NCF2, PDGFA and SRSF2 were upregulated in PCa patients compared to healthy controls and achieved good performance for PCa screening (Fig. 

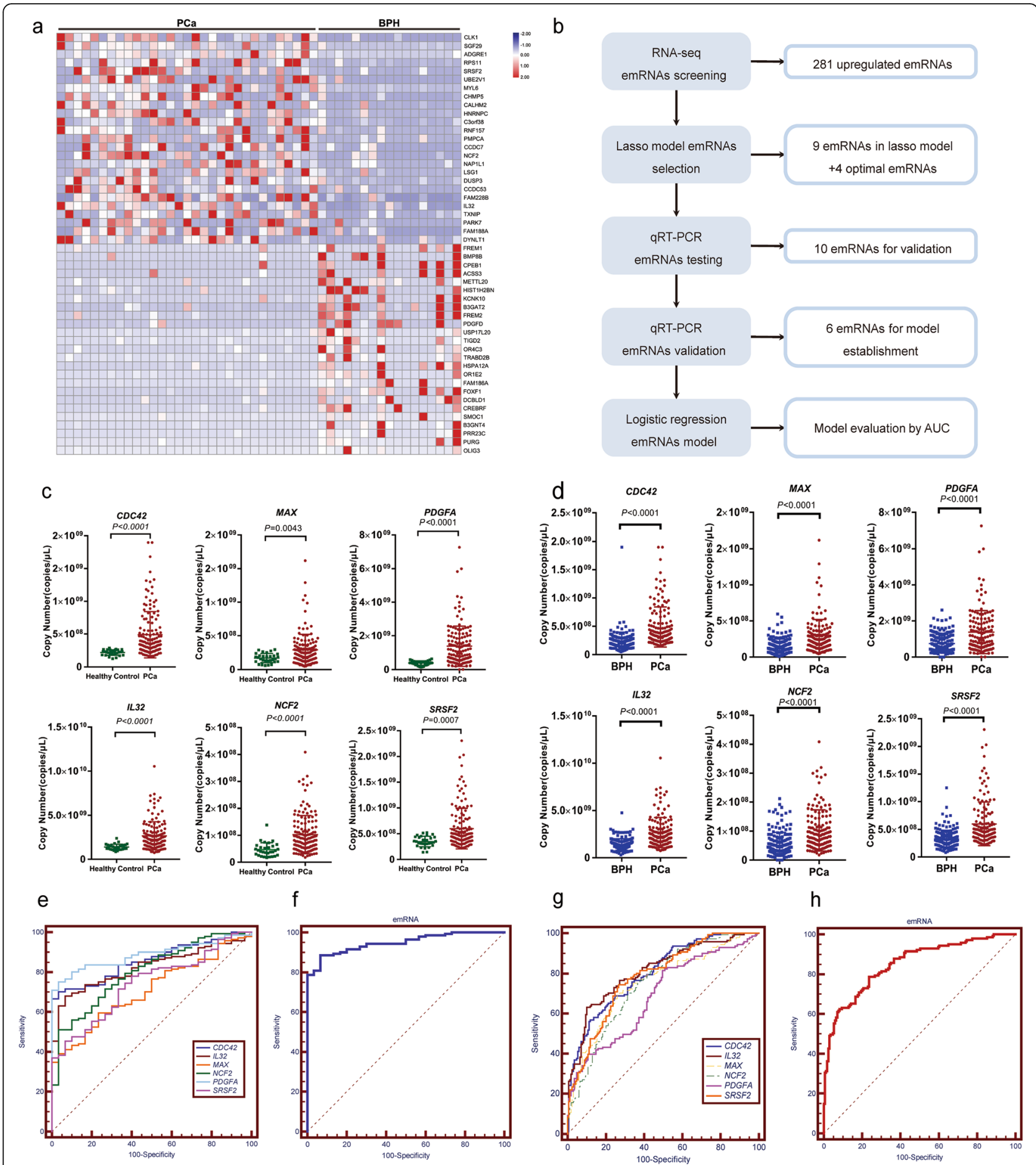

Fig. 2 Validation of circulating exosomal mRNAs (emRNAs) as novel biomarkers for PCa diagnosis. a, Heatmap demonstrates the significantly dysregulated emRNAs in PCa patients. Each column represents an individual sample, and each row represents an emRNA. b, Workflow of the validation of potential circulating emRNAs. c, The scatter plot shows that the expression levels of circulating emRNAs, including CDC42, IL32, MAX, NCF2, PDGFA and SRSF2, are upregulated in PCa patients $(n=141)$ compared to healthy controls $(n=30)$. $\mathbf{d}$, The scatter plot shows that the expression levels of circulating emRNAs, including CDC42, IL32, MAX, NCF2, PDGFA and SRSF2, are upregulated in PCa patients $(n=141)$ compared to patients with BPH (negative prostate biopsy, $n=170$ ). $\mathbf{e}$ and $\mathbf{g}$, ROC analysis shows the diagnostic performance of 6 mRNAs and the emRNAbased screening model (CDC42, IL32, MAX, NCF2, PDGFA and SRSF2; AUC: 0.948; $P<0.0001)$. $\mathbf{f}$ and $\mathbf{h}, \mathrm{ROC}$ analysis shows the diagnostic performance of 6 emRNAs and the emRNA-based diagnostic model (CDC42, IL32, MAX, NCF2, PDGFA and SRSF2) (AUC: 0.851; $P<0.0001$ ) 
2e). Furthermore, CDC42, IL32, MAX, NCF2, PDGFA and SRSF2 were upregulated in PCa compared to $\mathrm{BPH}$ (negative prostate biopsy) (Fig. 2d) and achieved good performance for $\mathrm{PCa}$ diagnosis (Fig. $2 \mathrm{~g}$ and Additional file 1: Table S4). We then used logistic regression analysis to establish an emRNA-based signature using the emRNAs described above. Receiver operating characteristic (ROC) analysis showed that the circulating emRNA-based screening signature (CDC42, IL32, MAX, NCF2, PDGFA and SRSF2) yielded an area under the ROC curve (AUC) of 0.948 in distinguishing PCa patients from healthy controls (Fig. 2f). More importantly, the circulating emRNA-based diagnostic signature $(C D C 42$, IL32, MAX, NCF2, PDGFA and SRSF2) showed great performance in predicting prostatic biopsy results (AUC: 0.851) (Fig. 2h). We also established the subtype signatures based on clinical and molecular parameters for the detection of $\mathrm{PCa}$ (Additional file 1: Figure S8, see details of Established the subtype signatures for the detection of $\mathrm{PCa}$ and Correlation analysis between emRNAs and the grade of PCa aggressiveness in Additional file 1). Furthermore, the source and potential importance of circulating emRNAs was demonstrated (Additional file 1: Figure S9-10, see details of The source of circulating emRNAs and The potential importance of the emRNAs in Additional file 1). Our results indicated that emRNA-based signatures could serve as a novel and promising method for the detection of PCa.

\section{Conclusion}

This is the first comprehensive study to investigate the characteristics of emRNA profiles in PCa patients and to evaluate the role of circulating emRNAs in the detection of $\mathrm{PCa}$. In this study, we developed an optimized emRNA detection strategy and identified novel emRNA signatures for PCa screening and diagnosis. These signatures could serve as novel noninvasive biomarkers for the improvement of $\mathrm{PCa}$ diagnosis.

\begin{abstract}
Abbreviations
mRNA: Messenger RNA; emRNA: Exosomal messenger RNA; PCa: Prostate cancer; BPH: Benign prostatic hyperplasia; ROC: Receiver operating characteristic; AUC: Area under the receiver operating characteristic curve; PSA: Prostate-specific antigen; HGPCa: High-grade prostate cancer; exRNA: Exosomal RNA; exLR: Extracellular long RNA; HCC: Hepatocellular carcinoma; PDAC: Pancreatic ductal adenocarcinoma; IGV: Integrative Genomics Viewer; RT-PCR: Reverse transcription polymerase chain reaction; qPCR: Quantitative polymerase chain reaction; LASSO: Least absolute shrinkage and selection operator.
\end{abstract}

\section{Supplementary Information}

The online version contains supplementary material available at https://doi. org/10.1186/s12943-021-01349-z.

Additional file 1: Figure S1. Quality control of exosomes isolation. Figure S2. Identificationc the existing forms of circulating emRNAs. Figure S3. Selection of potential diagnostic exosomes mRNA in the LASSO model. Figure S4. Optimized detection strategy for the detection of 13 PCa-associated emRNAs. Figure S5. Scatter plots of emRNA expression validation. Figure S6. Testing of previous reported reference genes.
Figure S7. Standard curve generated with real-time quantitative PCR. Figure S8. Established the subtype signatures for the detection of PCa. Figure S9. EmRNAs are derived from PCa and then released into the cell culture medium or circulation by packing into exosomes. Figure S10. The potential biological function of the emRNAs. Table S1. The list of dysregulated transcripts with varied expression between tissue and serum exosomes. Table S2. The list of primers and probes. Table S3. The list of upregulated emRNA in PCa. Table S4. Diagnosis performance of emRNAs. Table S5. Demographics of PCa patients and control participants for QC of exosome isolation. Table S6. Demographics of PCa patients and control participants for RNA-seq of their serum exosome. Table S7. Demographcs of PCa patients and control participants for dysregulated emRNAs validation. Table S8. Demographics of PCa patients and control participants for TaqMan qPCR testing. Table S9. Diagnosis performance of emRNAs in different PSA group. Table S10. Diagnosis performance of emRNAs in different ages. Table S11. Diagnosis performance of emRNAs in differentiating BPH and PCa with GS 6 from PCa with GS $\geq 7$. Identification the existing forms of circulating emRNAs. Optimized detection strategy for the detection of 13 PCa-associated emRNAs. Established the subtype signatures for the detection of PCa. Correlation analysis between emRNAs and the grade of PCa aggressiveness. The source of circulating emRNAs. The potential importance of the emRNAs. Methods.

\section{Acknowledgments}

We thank the National Natural Science Foundation of China (NSFC), Science and Technology Support Project in the field of biomedicine of Shanghai Science and Technology Action Plan, National Major Scientific and

Technological Special Project for Significant New Drug Development, Clinical Research Project of Shanghai Municipal Commission of Health and Family Planning, Shanghai Sailing Program, Jiangsu Provincial Medical Youth Talent, Precision Medicine Program of Second Military Medical University, Shanghai Rising-Star Program and Shanghai Health and Family Planning System Program.

\section{Authors' contributions}

F.W. conceptually designed the study, performed the experimental work, analyzed the data, and wrote the manuscript. J.J., R.C., L.Z. and Y.X. performed the experimental work, generated the data and the figures, and wrote the manuscript. J.J., R.C., Y.X., L.Z., Z.C., H.X., X.C., X.S., Y.Z. and J.H. collected the clinical samples and performed sample processing. L.Z., Z.C. and J.H. performed exosome purification and identification. H.X., X.C., X.S., Y.Z. performed emRNA quantification. F.W., J.L. and B.W. analyzed the RNAseq data and generated the figures. Y.W., T.Z. and X.W. contributed to discussing the hypothesis and data analysis and discussed the manuscript. B. W and B. Y critically reviewed the manuscript. F.W. led the project. The authors read and approved the final manuscript.

\section{Funding}

The work in this study is supported by grants from the National Natural Science Foundation of China (NSFC) (81902616 to F.W. and 81702514 to R.C.), Science and Technology Support Project in the field of biomedicine of Shanghai Science and Technology Action Plan (19441909200, F.W.), National Major Scientific and Technological Special Project for Significant New Drugs Development (2017ZX09304030, T.Z.), Clinical Research Project of Shanghai Municipal Commission of Health and Family Planning (20184Y0130, F.W.), Shanghai Sailing Program (19YF1447300, X.S.), Jiangsu Provincial Medical Youth Talent (QNRC2016739, X.W.) Precision Medicine Program of Second Military Medical University (2017JZ35, F.W.), National Natural Science Foundation of China (31971109, 31471390, Y.W.), Shanghai Rising-Star Program (17QA1405400, Y.W.) and Shanghai Health and Family Planning System Program (2017YQ028, Y.W.).

\section{Availability of data and materials}

All data associated with this study are presented in the paper and the Additional File 1. Materials and the online databases are indicated in the Methods section of the Additional File 1. Correspondence and requests for materials should be addressed to F.W. (wangbofengye@163.com). 


\section{Declaration}

\section{Ethics approval and consent to participate}

All of the subjects gave informed consent. This project was approved by the Clinical Research Ethics Committee of Shanghai Changhai Hospital.

\section{Consent for publication}

All authors gave consent for the publication of this study.

\section{Competing interests}

All authors state that there are no competing interests in this study.

\section{Author details}

'Department of Urology, Shanghai Changhai Hospital, Naval Medical University (Second Military Medical University), Shanghai, China. ${ }^{2}$ Division of Urology and Transplantation, Department of Surgery, Sichuan Academy of Medical Sciences and Sichuan Provincial People's Hospital and Hospital of The University of Electronic Science and Technology of China, Chengdu, Sichuan, China. ${ }^{3}$ Research Center of Developmental Biology, Naval Medical University (Second Military Medical University), Shanghai, China. ${ }^{4}$ Department of Urology, the First Affiliated Hospital of Soochow University, Suzhou, Jiangsu, China. ${ }^{5}$ Department of Pharmaceutical Sciences, College of Pharmacy and Pharmaceutical Sciences, Washington State University, Spokane, WA 99202, USA.

Received: 4 January 2021 Accepted: 15 March 2021

Published online: 30 March 2021

\section{References}

1. Bray F, Ferlay J, Soerjomataram I, Siegel RL, Torre LA, Jemal A. Global cancer statistics 2018: GLOBOCAN estimates of incidence and mortality worldwide for 36 cancers in 185 countries. CA Cancer J Clin. 2018;68:394-424.

2. Thompson IM, Pauler DK, Goodman PJ, Tangen CM, Lucia MS, Parnes HL, Minasian LM, Ford LG, Lippman SM, Crawford ED, Crowley JJ, Coltman CA Jr. Prevalence of prostate cancer among men with a prostate-specific antigen level < or =4.0 ng per milliliter. N Engl J Med. 2004;350(22):2239-46. https://doi.org/10.1056/NEJMoa031918.

3. Kalluri R, LeBleu VS. The biology, function, and biomedical applications of exosomes. Science. 2020;367(6478):eaau6977. https://doi.org/10.1126/ science.aau6977.

4. Wei Z, Batagov AO, Schinelli S, Wang J, Wang Y, El Fatimy R, Rabinovsky R, Balaj L, Chen CC, Hochberg F, et al. Coding and noncoding landscape of extracellular RNA released by human glioma stem cells. Nat Commun. 2017; 8(1):1145. https://doi.org/10.1038/s41467-017-01196-X.

5. Li Y, Zhao J, Yu S, Wang Z, He X, Su Y, Guo T, Sheng H, Chen J, Zheng Q, Li Y, Guo W, Cai X, Shi G, Wu J, Wang L, Wang P, He X, Huang S. Extracellular vesicles long RNA sequencing reveals abundant mRNA, circRNA, and IncRNA in human blood as potential biomarkers for Cancer diagnosis. Clin Chem. 2019;65(6):798-808. https://doi.org/10.1373/clinchem.2018.301291.

6. Yu S, Li Y, Liao Z, Wang Z, Wang Z, Li Y, Qian L, Zhao J, Zong H, Kang B, Zou WB, Chen K, He X, Meng Z, Chen Z, Huang S, Wang P. Plasma extracellular vesicle long RNA profiling identifies a diagnostic signature for the detection of pancreatic ductal adenocarcinoma. Gut. 2020;69(3):540-50. https://doi.org/10.1136/gutjnl-2019-318860.

7. Das $S$, Extracellular RNA Communication Consortium, Ansel KM, Bitzer M, Breakefield XO, Charest A, Galas DJ, Gerstein MB, Gupta M, Milosavljevic A, et al. The extracellular RNA communication consortium: establishing foundational knowledge and Technologies for Extracellular RNA research. Cell. 2019;177(2):231-42. https://doi.org/10.1016/j.cell.2019.03.023.

8. Rodríguez M, Bajo-Santos C, Hessvik NP, Lorenz S, Fromm B, Berge V, Sandvig K, Linē A, Llorente A. Identification of non-invasive miRNAs biomarkers for prostate cancer by deep sequencing analysis of urinary exosomes. Mol Cancer. 2017;16(1):156. https://doi.org/10.1186/s12943-0170726-4.

9. Huang X, Yuan T, Liang M, Du M, Xia S, Dittmar R, Wang D, See W, Costello BA, Quevedo F, et al. Exosomal miR-1290 and miR-375 as prognostic markers in castration-resistant prostate cancer. Eur Urol. 2015;67(1):33-41. https://doi.org/10.1016/j.eururo.2014.07.035.

10. McKiernan J, Donovan MJ, O'Neill V, Bentink S, Noerholm M, Belzer S, Skog J, Kattan MW, Partin A, Andriole G, et al. A novel urine exosome gene expression assay to predict high-grade prostate Cancer at initial biopsy. JAMA Oncol. 2016;2(7):882-9. https://doi.org/10.1001/jamaoncol.2016.0097.

11. McKiernan J, Donovan MJ, Margolis E, Partin A, Carter B, Brown G, Torkler P, Noerholm M, Skog J, Shore N, Andriole G, Thompson I, Carroll P. A prospective adaptive utility trial to validate performance of a novel urine exosome gene expression assay to predict high-grade prostate Cancer in patients with prostate-specific antigen 2-10 ng/ml at initial biopsy. Eur Urol. 2018;74(6):731-8. https://doi.org/10.1016/j.eururo.2018.08.019.

12. Hinger SA, Cha DJ, Franklin JL, Higginbotham JN, Dou Y, Ping J, Shu L, Prasad N, Levy S, Zhang B, Liu Q, Weaver AM, Coffey RJ, Patton JG. Diverse long RNAs are differentially sorted into extracellular vesicles secreted by colorectal Cancer cells. Cell Rep. 2018;25(3):715-25. https://doi.org/10.1016/j. celrep.2018.09.054

\section{Publisher's Note}

Springer Nature remains neutral with regard to jurisdictional claims in published maps and institutional affiliations.

Ready to submit your research? Choose BMC and benefit from:

- fast, convenient online submission

- thorough peer review by experienced researchers in your field

- rapid publication on acceptance

- support for research data, including large and complex data types

- gold Open Access which fosters wider collaboration and increased citations

- maximum visibility for your research: over $100 \mathrm{M}$ website views per year

At $\mathrm{BMC}$, research is always in progress.

Learn more biomedcentral.com/submissions 